\title{
Prática Baseada em Evidências
} em Psicologia e a História da Busca pelas Provas Empíricas da Eficácia das Psicoterapias*

Evidence-Based Practice in Psychology and the History of the Search for Empirical Proof on the Efficacy of Psychotherapies

La Práctica Basada en la Evidencia en la Psicología y la Historia de Búsqueda de las Pruebas Empíricas sobre la Eficacia de las Psicoterapias

Jan Luiz Leonardi \& Sonia Beatriz Meyer

Universidade de São Paulo

http://dx.doi.org/10.1590/1982-3703001552014

(cc) BY

* Este artigo é parte da tese de doutorado do primeiro autor (bolsista CAPES, Processo $\mathrm{n}^{\circ}$ 13.1.00056.47.7), orientada pela segunda autora (bolsista de produtividade em pesquisa do $\mathrm{CNPq}^{-}$Nível 2, Processo n 303716/2012-6) 
Resumo: Tradicionalmente, a escolha pelo tipo de intervenção psicoterápica para diferentes quadros clínicos depende fundamentalmente da experiência profissional do terapeuta e de sua predileção por determinadas estratégias clínicas. Todavia, esse cenário tem se modificado no contexto da prática baseada em evidências. Apesar do extenso e acalorado debate na literatura internacional da Psicologia nos últimos anos, o tema da prática baseada em evidências tem sido pouco discutido na Psicologia brasileira. Em vista disso, este artigo tem por objetivo apresentar o paradigma de prática baseada em evidências em Psicologia sob uma perspectiva histórica, enfatizando o embate entre os diferentes posicionamentos sobre o assunto e os esforços da American Psychological Association em elaborar um modelo representativo das diversas perspectivas teóricas, metodológicas, conceituais e práticas com o intuito de preencher a lacuna entre ciência e prática ainda existente na Psicologia Clínica. Espera-se que este artigo possa contribuir para uma maior participação da Psicologia brasileira nesse movimento.

Palavras-chave: Prática Baseada em Evidências. Psicologia Clínica. Psicoterapia.

Abstract: Traditionally, the choice of the type of psychotherapeutic intervention for various clinical conditions fundamentally depends on the professional experience of the therapist and his/her predilection for certain clinical strategies. However, this scenario has been changing in the context of evidence-based practice. Despite the extensive and heated debate in international psychology literature over the past few years, evidence-based practice has been scarcely discussed in Brazilian Psychology. Hence, this article aims at introducing the paradigm of evidence-based practice in psychology from a historical perspective, with particular emphasis on the clash between the different positions on the issue. It also focuses on the efforts of the American Psychological Association to design a model that is representative of various theoretical, methodological, conceptual, and practical perspectives in order to bridge the gap between science and practice that still exists in clinical Psychology. This article seeks to contribute to a greater participation of Brazilian psychology in this movement.

Keywords: Evidence-Based Practice. Evidence-Based Psychology. Clinical Psychology. Psychotherapy.

Resumen: Tradicionalmente, la elección del tipo de intervención psicoterapéutica para diversas condiciones clínicas depende fundamentalmente de la experiencia profesional del terapeuta y de su predilección por determinadas estrategias clínicas. Sin embargo, este escenario ha ido cambiando en el contexto de la práctica basada en la evidencia. A pesar del debate extenso y arduo en la literatura internacional de la psicología en los últimos años, el tema de la práctica basada en la evidencia ha sido poco discutido en la Psicología brasileña. En vista de ello, este artículo tiene como objetivo presentar el paradigma de la práctica basada en la evidencia en la psicología desde una perspectiva histórica, haciendo hincapié en el choque entre las diferentes posiciones sobre el tema y los esfuerzos de la American Psychological Association para desarrollar un modelo representativo de las distintas perspectivas teóricas, metodológicas, conceptuales y prácticas con el fin de rellenar la brecha entre la ciencia y la práctica aún vigente en Psicología clínica. Se espera que este artículo permita contribuir para una mayor participación de la psicología brasileña en este movimiento.

Palabras clave: Práctica Basada en la Evidencia. Psicologia Clinica. Psicoterapia.

\section{Introdução}

Tradicionalmente, a escolha pelo tipo de intervenção psicoterápica para diferentes quadros clínicos depende fundamentalmente da experiência profissional do terapeuta e de sua predileção por determinadas estratégias clínicas (Gaudiano, Brown \& Miller, 2011; Morrow-Bradley \& Elliott, 1986). Esse cenário, entretanto, tem se modificado no contexto da prática baseada em evidências, cuja premissa 
é a de que a conduta do psicólogo deve ser fundamentada em dados empíricos. Embora tenha sido desenvolvida originalmente na década de 1990 na medicina (cf. Guyatt et al., 1992), a prática baseada em evidências vem recebendo um crescente interesse na Psicologia (Goodheart, Kazdin \& Sternberg, 2006; Norcross, Beutler \& Levant, 2006) e em outras ciências da saúde, tais como Fisioterapia (Filippin \& Wagner, 2008) e Odontologia (Richards, 2008).

O advento da prática baseada em evidências foi influenciado por um conjunto de fatores científicos, sociais, econômicos e políticos, tais como interesse de pesquisadores, clínicos e associações profissionais em comprovar os resultados dos serviços prestados pela categoria, demanda dos consumidores em assegurar a qualidade dos serviços recebidos, empenho das agências governamentais em proteger os direitos dos consumidores, interesse dos planos de saúde em maximizar a relação custo-benefício dos tratamentos, entre outros (Beutler, 1998).

A prática baseada em evidências é, atualmente, um dos assuntos mais discutidos no cenário internacional da Psicologia. Com raras exceções, há consenso de que o status da psicoterapia e a autonomia profissional do psicólogo clínico dependem fortemente de práticas terapêuticas que estejam solidamente embasadas em evidências empíricas. Todavia, a compreensão do que constituem essas evidências e como elas deveriam ser produzidas, avaliadas e aplicadas é alvo de uma enormidade de controvérsias (Goodheart et al., 2006; Norcross et al., 2006).

Apesar do extenso e acalorado debate na literatura internacional da Psicologia nos últimos 20 anos, o tema da prática baseada em evidências tem sido pouco debatido no cenário da Psicologia brasileira e são raras as publicações em língua portuguesa (algumas exceções são Baptista, 2010; Melnik \& Atallah, 2011; Melnik, Souza \& Carvalho, 2014; Silva \& Otta, 2013). Em vista disso, este artigo tem por objetivo apresentar o desenvolvimento do paradigma de prática baseada em evidências em Psicologia (PBEP) sob uma perspectiva histórica, enfatizando o embate entre os diferentes posicionamentos sobre o tema e os esforços da American Psychological Association (APA) em elaborar um modelo representativo das diversas perspectivas teóricas, metodológicas, conceituais e práticas.

\section{Desenvolvimento histórico da Prática Baseada em Evidências em Psicologia}

Em 1952, o psicólogo alemão Hans Eysenck publicou uma revisão de 19 estudos empíricos de psicoterapia de sua época, nos quais a única medida de resultado disponível era o registro do terapeuta em termos de cura ou muita melhora, melhora, pouca melhora e nenhuma melhora, e concluiu que nenhuma modalidade de intervenção psicoterápica era mais efetiva para a melhora do cliente do que a mera passagem do tempo. O autor, ao resumir os dados analisados por ele, atesta que

eles falham em provar que a psicoterapia, Freudiana ou não, facilita a recuperação de pacientes neuróticos. Eles mostram que aproximadamente dois terços de um grupo de pacientes neuróticos irá se recuperar ou melhorar de forma significativa cerca de dois anos após o início de sua doença, sejam eles tratados por meio da psicoterapia ou não. Esse cenário parece ser notavelmente estável de uma investigação para outra, a despeito do tipo de paciente tratado, medida de recuperação utilizada ou método de terapia empregado (Eysenck, 1952, pp. 322-323).

As conclusões de Eysenck (1952) foram inquietantes e levaram a diferentes tipos de reação. Alguns terapeutas ignoraram a publicação de Eysenck, defendendo que a prática da psicoterapia era importante. $\mathrm{O}$ argumento era que "todo mundo sabe que terapia funciona" (Barlow, Boswell \& Thompson-Hollands, 2013). Um exemplo que ilustra essa posição é a afirmação de Strupp (1964), que mais tarde se tornaria um proeminente pesquisador da psicoterapia, de que "observações clínicas documentam amplamente que muitos pacientes se beneficiam de uma relação interpessoal com um profissional quando estão perturbados por dificuldades da vida e procuram ajuda. 
Argumentar o contrário é simplesmente fechar os olhos para os fatos" (p. 101).

Outras reações por parte da comunidade foram o questionamento da viabilidade e utilidade da prática psicoterápica para o campo da saúde mental, por um lado, e a assertiva de que a pesquisa em psicoterapia não poderia produzir nada de relevante para os terapeutas, por outro. Carl Rogers, por exemplo, argumentava que a pesquisa em psicoterapia não exercia nenhum impacto em sua prática clínica e que, portanto, poderia ser abandonada (Barlow et al., 2013).

O principal efeito do trabalho de Eysenck (1952), entretanto, foi estimular a busca pela demonstração empírica dos resultados das psicoterapias (Barlow et al., 2013). Revisões de pesquisas clínicas realizadas nas décadas de 1950, 1960 e 1970 surgiram na segunda metade da década de 1970 e, em sua maioria, demonstravam que a psicoterapia é altamente efetiva e que não existem diferenças significativas entre as várias modalidades. Por exemplo, em 1975, Luborsky, Singer e Luborsky analisaram 105 pesquisas que compararam o efeito de diferentes modalidades de psicoterapia entre si, tais como psicanálise, terapia centrada no cliente, terapia comportamental e psicodrama, além de comparações com tratamentos medicamentosos e com grupos-controle. Os autores concluíram que "a maioria dos estudos comparativos das diferentes formas de psicoterapia revelaram diferenças insignificantes nas proporções de pacientes que melhoraram ao final da psicoterapia" (p. 1003). Em outras palavras, todos se beneficiaram da terapia, a despeito da teoria ou técnica empregada.

Na mesma direção, Smith, Glass e Miller (1980) revisaram 475 estudos que compararam o efeito da psicoterapia com grupos-controle. Os autores encontraram um tamanho de efeito (medida que indica quanta mudança ocorreu na variável dependente em relação à introdução da variável independente) de 0.85 , ou seja, uma diferença de $85 \%$ entre a média dos indivíduos que receberam intervenção psicoterápica em relação ao grupo que não recebeu. Embora essa descoberta tenha estabelecido a eficácia da psicoterapia, Strupp e Howard (1992) fazem alguns alertas sobre a interpretação dos dados oferecida por Smith et al. (1980):
parece justo apontar que $50 \%$ do grupo sem tratamento teria tido um resultado igual ou melhor do que a pessoa média não tratada (i.e., numa distribuição normal, metade dos sujeitos irá cair na média ou acima da média). Em outras palavras, a psicoterapia adiciona 30\% a esse critério estatístico. Além disso, seus resultados indicam que $20 \%$ das pessoas tratadas presumivelmente teriam um resultado igual ou menor do que a pessoa média não tratada [ou seja, a psicoterapia seria prejudicial para 20\% dos indivíduos]. (p. 312)

Com base nos resultados de Luborsky et al. (1975) e de Smith et al. (1980), a eficácia das psicoterapias foi atribuída a características que estão presentes nas mais diversas intervenções terapêuticas, os chamados fatores comuns, que incluem qualidades do terapeuta (e.g., empatia e aceitação), características do cliente (e.g., expectativa de melhorar com a terapia) e relação terapêutica, esta considerada a principal variável causal do sucesso da intervenção (DeRubeis, Brotman \& Gibbons, 2005). De acordo com os defensores dos fatores comuns, as especificidades de cada tratamento seriam relativamente pouco importantes para o resultado final do processo terapêutico (Wampold, 2010). Nesse sentido, Luborsky et al. (1975) chegam a afirmar que "de agora em diante nós deveríamos parar de dar atenção à forma do tratamento ao indicar pacientes para a psicoterapia" (p. 1005). A ideia de que todas as formas de terapia funcionam igualmente bem ficou conhecida na literatura como o "veredito do pássaro Dodô", em alusão a uma passagem de Alice no País das Maravilhas: "ao final, o Dodô disse: todos venceram e todos merecem prêmios".

Muitos pesquisadores (e.g., Beutler, 1979; Kazdin \& Bass, 1989), em sua maioria de orientação cognitivo-comportamental, suspeitavam da veracidade do veredito do pássaro Dodô e argumentavam que os principais responsáveis pela mudança terapêutica seriam os fatores específicos - procedimentos, 
técnicas e estratégias - presentes em cada modalidade de terapia. O paradigma dos fatores específicos é composto de cinco componentes: (1) há um comportamento-alvo, queixa ou transtorno a ser tratado (e.g., fobia de barata); (2) há uma explicação teórica sobre a origem do problema (e.g., condicionamento respondente); (3) há mecanismos de mudança consistentes com determinada abordagem teórica (e.g., extinção respondente); (4) esses mecanismos de mudança indicam uma terapêutica a ser empregada (e.g., dessensibilização sistemática); (5) os resultados (e.g., cura da fobia) são atribuídos à terapêutica empregada, ou seja, a um fator específico da intervenção.

Com o intuito de comprovar o papel dos fatores específicos, a Divisão 12 da APA (Psicologia Clínica) instaurou, em 1993, uma força-tarefa composta por psicólogos de diferentes abordagens teóricas, cujos objetivos eram definir e identificar tratamentos empiricamente validados (expressão posteriormente substituída por tratamentos empiricamente sustentados, com o argumento de que o termo validado talvez sugerisse erroneamente que a pesquisa de determinada intervenção estava completa e era definitiva), propor diretrizes de formação para futuros terapeutas e de treinamento contínuo para os profissionais em exercício, disseminar os tratamentos empiricamente sustentados para os planos de saúde e informar a população sobre a eficácia da psicoterapia (Chambless, 1993).

Chambless et al. (1998) explicam o processo utilizado na busca por tratamentos empiricamente sustentados: inicialmente, a força-tarefa pediu indicações a seus pares por meio de diversos canais de comunicação (publicações oficiais da APA e e-mails da Society for Psychotherapy Research e da Society for a Science of Clinical Psychology, por exemplo) e conduziu revisões de literatura em periódicos que publicam pesquisas empíricas de psicoterapia. Uma vez identificados os potenciais candidatos a tratamentos empiricamente sustentados, um revisor recebia a incumbência de avaliar a eficácia de um tratamento em particular. Quando a evidência era ambígua, o revisor tinha a responsabilidade de determinar se a preponderância era positiva ou negativa, atribuindo maior ou menor crédito aos dados de um estudo a depender de sua qualidade metodológica; caso a evidência fosse negativa, o tratamento não entrava na lista. Posteriormente, o revisor apresentava suas conclusões a um grupo maior e pontos de discordância eram debatidos até que um consenso fosse alcançado. É importante observar que a forçatarefa previa que novos tratamentos poderiam ser adicionados à lista e que tratamentos já listados poderiam ser removidos ou terem sua classificação alterada à medida que novas evidências fossem produzidas.

Nesse trabalho, a força-tarefa considerou apenas os tratamentos que estavam descritos em manuais, sob a premissa de que isso contribuiria para delimitar o que a intervenção realmente incluiu e, portanto, o que realmente foi sustentado (Chambless et al., 1996; Sanderson \& Woody, 1995). Além disso, a sustentação empírica dos tratamentos foi avaliada de acordo com problemas clínicos específicos, tais como transtorno do pânico, transtorno obsessivo-compulsivo, fobias, depressão, estresse, disfunções sexuais, problemas de saúde (síndrome do cólon irritável, dor crônica), entre outros. Desse modo, uma modalidade de terapia não seria simplesmente classificada como um tratamento empiricamente sustentado, mas sim como um tratamento empiricamente sustentado para transtorno depressivo maior ou um tratamento empiricamente sustentado para transtorno de personalidade borderline, por exemplo.

Um tratamento era considerado empiricamente sustentado se houvesse: (1) dois ou mais ensaios clínicos randomizados conduzidos por diferentes pesquisadores que demonstrassem que aquele tratamento era superior a placebo ou a outras intervenções psicoterápicas ou que eram equivalentes a um tratamento já estabelecido em estudos estatisticamente significantes; (2) nove ou mais experimentos de caso único conduzidos por diferentes pesquisadores que demonstrassem que aquele tratamento era superior a placebo ou a outros tratamentos já estabelecidos. Um tratamento era considerado provavelmente 
eficaz quando havia: (1) dois estudos que demonstrassem que aquele tratamento era superior a um grupo sem tratamento ou (2) três ou mais experimentos de caso único que demonstrassem que aquele tratamento era superior a placebo ou a outros tratamentos. Um tratamento também era listado como provavelmente eficaz quando atendia aos critérios de tratamento empiricamente sustentado, mas os estudos que o sustentavam tinham sido realizados por um mesmo grupo de pesquisadores.

Em 1995, após a análise de diversos grupos de discussão acerca de seu conteúdo, o relatório da força-tarefa da Divisão 12 da APA foi publicado no boletim The Clinical Psychologist, no qual foram listados 18 tratamentos empiricamente sustentados e sete provavelmente eficazes, com a observação de que os tratamentos que não haviam sido incluídos nessas duas categorias deveriam ser considerados experimentais (Task Force on Promotion and Dissemination of Psychological Procedures, 1995). Uma nova atualização da lista foi publicada pela Divisão 12 em 1998, na qual foram relacionados 16 tratamentos empiricamente sustentados e 55 tratamentos provavelmente eficazes, além de uma lista atualizada dos manuais que descrevem as etapas e os procedimentos dos tratamentos empiricamente sustentados e de um conjunto de informações sobre oportunidades de formação e treinamento nessas psicoterapias (Chambless et al., 1998; Woody \& Sanderson, 1998).

Em 1999, com o intuito de dar continuidade à identificação, formação e disseminação de tratamentos empiricamente sustentados, a Divisão 12 formou um comitê permanente que publica atualizações constantes sobre a eficácia de intervenções psicológicas, disponíveis em seu website (http://www. psychologicaltreatments.org). Desde a formação da força-tarefa em 1993, o trabalho da Divisão 12 resultou no desenvolvimento de critérios para a identificação da sustentação empírica de diversas intervenções psicológicas, na identificação de pesquisas de resultado, na publicação de listas que relacionam os tratamentos empiricamente sustentados para diversos diagnósticos, na elaboração de manuais de tratamento e no desenvolvimento de recursos de formação e treinamento nos tratamentos listados (Sanderson, 2003).

Apesar dessas conquistas, o produto da força-tarefa da Divisão 12 foi contestado por diversos pesquisadores e terapeutas, por várias razões. Uma delas diz respeito à inclusão somente de pesquisas cujos participantes preenchiam os critérios diagnósticos para alguma psicopatologia descrita no Manual Diagnóstico e Estatístico de Transtornos Mentais (DSM; American Psychiatric Association, 2013). Alguns autores (cf. Reed, Kihlstrom \& Messer, 2006) argumentam que os diagnósticos não capturam as particularidades que os clientes levam para o consultório e nem os contextos em que os problemas surgiram e se mantêm. Outros (e.g., Bohart, O'Hara \& Leitner, 1998) defendem que os diagnósticos não são necessários para descrever os clientes e que rotular um grupo de indivíduos apenas por compartilharem algumas características não os torna semeIhantes; ao contrário, cada cliente deveria ser visto como único e, portanto, receber um tratamento totalmente individualizado.

Outros autores explicam que boa parte dos clientes costuma apresentar sintomas de ansiedade, depressão, uso de drogas, alterações de personalidade, etc., que estão abaixo do limiar para fechar um diagnóstico completo, e que muitos outros buscam a terapia para resolver questões que sequer estão descritas no DSM, como insatisfação com o emprego, dúvida sobre a permanência ou não em um casamento, dificuldade em se envolver em relacionamentos afetivos, entre outros (Fava \& Mangelli, 2001; Stirman, DeRubeis, Crits-Christoph, \& Brody, 2003; Westen \& Arkowitz-Westen, 1998).

Outra razão de insatisfação por parte de clínicos e pesquisadores foi a inclusão somente de tratamentos manualizados. Conforme mencionado, os manuais de tratamento apresentam protocolos padronizados de intervenção que devem ser seguidos pelo terapeuta frente a problemas clínicos específicos. Alguns 
manuais prescrevem o número de sessões requeridas e os procedimentos que devem ser aplicados em cada uma das sessões, enquanto outros apenas fornecem diretrizes flexíveis para a conduta terapêutica. A Divisão 12 entende que a descrição do tratamento na forma de manual é necessária para haver uma definição clara da intervenção (a variável independente em questão) e, também, porque procedimentos muito diferentes podem ser incluídos sob a mesma rubrica, como, por exemplo, terapia cognitivo-comportamental ou psicoterapia psicodinâmica (Chambless \& Ollendick, 2001). De acordo com Addis, Wade e Hatgis (1999), a reação negativa à manualização deve-se a um conjunto de preocupações por parte de clínicos e pesquisadores de que seguir o manual atrapalharia o desenvolvimento de uma boa relação terapêutica, ignoraria as diferenças individuais, não atenderia às necessidades de clientes com múltiplos problemas e/ou diagnósticos, ameaçaria a independência, espontaneidade e criatividade do clínico e retardaria o desenvolvimento de novas teorias e de intervenções alternativas.

Em contrapartida, defensores da manualização (e.g., Addis \& Cardemil, 2006; Chambless \& Ollendick, 2001) argumentam que a função dos manuais não é substituir a inventividade, flexibilidade ou sensibilidade do terapeuta, mas apenas descrever os ingredientes ativos de determinado tipo de psicoterapia. Para eles, os manuais são uma boa maneira de descrever as características conceituais, estruturais e técnicas de determinado tratamento sem deixar de lado a relação terapêutica, além de proverem um guia para os terapeutas monitorarem sua adesão a um conjunto de intervenções previamente testadas. Dessa forma, os manuais de tratamento ofereceriam vários benefícios para uma prática embasada em evidências.

Outro alvo de crítica ao trabalho da Divisão 12 refere-se à avaliação dos tratamentos por meio de pesquisas cujo método empregado foi o ensaio clínico randomizado, cujos alcances e limites para a pesquisa em psicoterapia estão fora do escopo do presente artigo (cf. Starcevic, 2003; Stiles et al., 2006).
A força-tarefa recebeu três grupos de críticas por considerar o ensaio clínico randomizado o padrão-ouro para a produção de evidências (Chambless \& Ollendick, 2001): (1) os estudos de grupo, essencialmente quantitativos, seriam pobres para o campo da psicoterapia, sendo a pesquisa qualitativa a mais apropriada; (2) a abordagem cognitivo-comportamental teria levado vantagem na avaliação porque foram muito mais pesquisadas com esse tipo de método do que todas as outras abordagens e, assim, teria sido injustamente considerada mais eficaz; (3) o fato de um tratamento ser eficaz (i.e., demonstrar resultados satisfatórios em condições controladas de pesquisa - validade interna) não garantiria que ele seja efetivo (i.e., apresentar resultados satisfatórios no contexto da oferta real de serviços de psicoterapia - validade externa), sobretudo porque os critérios de seleção dos participantes das pesquisas anulariam a compatibilidade entre estes e os clientes que procuram a clínica particular (cf. Westen, Stirman \& DeRubeis, 2006).

As críticas direcionadas ao trabalho da Divisão 12 foram o ponto de partida para o surgimento de forças-tarefas de outras divisões da APA, cuja intenção era corrigir e complementar possíveis omissões na avaliação das intervenções psicoterápicas. Em 1999, uma força-tarefa foi formada pela Divisão 29 da APA (Psicoterapia) com o objetivo de identificar, operacionalizar e disseminar informações sobre relações terapêuticas empiricamente sustentadas. A equipe conduziu 24 meta-análises de um corpo vasto de pesquisas quantitativas e qualitativas que deu origem a uma listagem de elementos da relação terapêutica (aliança de trabalho, empatia, etc.) e de características do cliente (estilo de enfrentamento, expectativas, etc.) que contribuiriam de forma consistente para o resultado da terapia. O produto final desse trabalho foi publicado no livro Psychotherapy Relationships That Work: Therapist Contributions and Responsiveness to Patient Needs (Norcross, 2002).

A tensão entre as forças-tarefa das diferentes divisões da APA intensificou o debate sobre o que deveria ser empiricamente validado na 
pesquisa em psicoterapia - fatores comuns ou fatores específicos. A abordagem dos fatores comuns foi fortalecida pelo trabalho de Luborsky et al. (2002), que examinaram 17 meta-análises sobre o efeito de diferentes psicoterapias para depressão, transtornos de ansiedade e neuroses mistas, tendo encontrado um tamanho de efeito de 0.20 , o que indica pouca diferença entre as intervenções. Como já apontado, os defensores do veredito do pássaro Dodô argumentam que, uma vez que todas as formas de psicoterapia funcionam igualmente bem e que em todas elas o terapeuta procura estabelecer a melhor relação terapêutica possível, os resultados da intervenção provavelmente são fortemente determinados por esse fator comum. Nesse contexto, Wampold (2010) chega até mesmo a sugerir que "ensaios clínicos comparando dois tratamentos deveriam ser descontinuados" (p. 71).

Em contraposição, diversos autores (e.g., Beutler, 2002; Chambless, 2002; DeRubeis et al., 2005; Kazdin, 2005; Rounsaville \& Carroll, 2002) questionaram as conclusões dos defensores dos fatores comuns, tanto por razões metodológicas quanto empíricas. Do ponto de vista metodológico, é um erro colocar clientes com diferentes características diagnósticas e/ou queixas clínicas em um mesmo grupo, na medida em que as diversas idiossincrasias dos participantes poderiam afetar diferencialmente suas respostas aos tratamentos. Nesse sentido, agrupar mais de 100 tipos diferentes de clientes em um único grupo, como fizeram Luborsky et al. (1975; 2002), pode mascarar os efeitos dos fatores específicos ao forçar uma homogeneidade inexistente entre os participantes (Beutler, 2002). Para DeRubeis et al. (2005), perguntar se determinada abordagem terapêutica é, na média, superior a alguma outra para todas as queixas clínicas "é o mesmo que perguntar se insulina ou antibiótico é melhor, sem saber a condição na qual esses tratamentos serão administrados" (p. 175). Na perspectiva desses autores, questões genéricas como essa comprometem os resultados obtidos e induzem à conclusão equivocada de que o veredito do pássaro Dodô está correto.
Outro erro metodológico cometido nas meta-análises dos defensores dos fatores comuns foi agrupar as centenas de psicoterapias em alguns poucos rótulos (Beutler, 2002). Por exemplo, na revisão de Luborsky et al. (2002), diferentes escolas teóricas foram tratadas como funcionalmente equivalentes: abordagens distintas como sistêmica, transpessoal, existencial e centrada na pessoa foram agrupadas sob a rubrica de "dinâmicas". Um erro semelhante cometido pelos autores foi colocar os diversos tratamentos farmacológicos envolvidos nas pesquisas revisadas em um só grupo. Segundo Beutler (2002), a decisão de ignorar o tipo de medicação utilizada e a diversidade de efeitos desses medicamentos para diferentes sintomas demonstra uma enorme depreciação da farmacologia. Tendo em vista a heterogeneidade de diagnósticos da amostra, é muito provável que o grupo nomeado de tratamento medicamentoso tenha abarcado inibidores seletivos da recaptação de serotonina, antidepressivos tricíclicos, benzodiazepínicos, anticonvulsivantes, estimulantes e antipsicóticos, cujos efeitos variam enormemente a depender dos problemas clínicos em investigação.

Outro problema diz respeito à impossibilidade de estabelecer o papel causal da relação terapêutica na melhora observada no processo de psicoterapia, visto que os mais de 2.000 estudos sobre o tema mostram apenas correlação entre a qualidade da relação e a intensidade da melhora (Kazdin, 2005). Uma implicação disso é que a equivalência entre diversas modalidades de psicoterapia revelada nas revisões de literatura (e.g. Luborsky et al., 1975; 2002; Smith et al., 1980) não precisa ser, necessariamente, explicada por meio dos fatores comuns. Para DeRubeis et al. (2005), é possível que intervenções diferentes produzam resultados semelhantes por meio de fatores específicos distintos. Um exemplo provém das pesquisas que comparam tratamentos psicológicos com tratamentos farmacológicos. Em muitos casos, psicoterapia e medicamento promovem mudanças similares no sistema nervoso do indivíduo (cf. Cozolino, 2010), supostamente por meio de mecanismos de ação distintos. Em suma, "a literatura de aliança [terapêutica], pelo menos em seu estado atual, assim como acontece 
com a literatura de comparação de resultados, não favorece os fatores comuns como uma explicação para a mudança terapêutica" (Kazdin, 2005, p. 186).

Além dos questionamentos metodológicos, há um vasto conjunto de evidências empíricas que demonstram a superioridade de certos tratamentos psicológicos para determinados problemas clínicos. Alguns exemplos são exposição com prevenção de respostas para transtorno obsessivocompulsivo (cf. Williams, Powers \& Foa, 2012), terapia cognitivo-comportamental para transtorno do pânico (cf. Koerner, Vorstenbosch \& Antony, 2012) e terapia comportamental dialética para transtorno de personalidade borderline (cf. Sneed, Fertuck, Kanellopoulos \& Culang-Reinlieb, 2012). Para Beutler (2002), exemplos como esses deveriam ser interpretados como prova de que fatores específicos podem fazer toda a diferença. Ademais, existem evidências de que certas intervenções podem ser prejudiciais, como o gerenciamento de estresse de incidente crítico, a terapia de comunicação facilitada e o programa educacional de resistência às drogas e à violência, entre outras (Lilienfeld, 2007).

Em meio ao embate entre os defensores dos fatores comuns e os defensores dos fatores específicos, uma força-tarefa foi constituída em 2002 pela Society for Psychotherapy Research em conjunto com a Divisão 12 da APA, cuja premissa era a de que todos os fatores envolvidos no processo psicoterápico - qualidades do terapeuta, características do cliente, relação terapêutica e técnicas específicas - estariam relacionados ao resultado e, portanto, deveriam ser levados em consideração. Nesse sentido, o esforço conjunto da Society for Psychotherapy Research com a Divisão 12 pretendia, de certa forma, integrar os trabalhos anteriores da Divisão 12 e da Divisão 29. As duas questões que orientaram essa nova força-tarefa foram: (1) o que se sabe sobre a natureza dos participantes (terapeuta e cliente), da relação terapêutica e dos procedimentos no que diz respeito à eficácia da intervenção? (2) como as variáveis relacionadas aos participantes, à relação terapêutica e aos procedimentos trabalham a favor, contra e entre si na terapia? (Beutler \& Johannsen, 2006; Castonguay \& Beutler, 2006a; 2006b). Para responder a essas perguntas, diversos pesquisadores revisaram, entre outros estudos, a literatura de pesquisa na qual as forças-tarefa da Divisão 12 e da Divisão 29 se basearam, levando em conta quatro grupos de problemas clínicos: transtornos disfóricos, transtornos de ansiedade, transtornos de personalidade e transtornos relacionados ao uso de substâncias. Esse trabalho de revisão, publicado em 2006 no livro Principles of Therapeutic Change That Work (Castonguay \& Beutler, 2006b), deu origem à formulação de princípios de mudança terapêutica, que "são mais gerais do que uma descrição de técnicas e mais específicos do que formulações teóricas" (Castonguay \& Beutler, 2006b, p. 5). Tais princípios supostamente poderiam ser aplicados em qualquer modalidade de psicoterapia, já que abarcam variáveis relacionadas ao terapeuta, cliente, relação terapêutica e técnicas extraídas da literatura empírica que foram apresentadas sem ligação com qualquer teoria (Beutler \& Johannsen, 2006; Castonguay \& Beutler, 2006a; 2006b). Alguns exemplos de princípios de mudança terapêutica são: "a motivação do cliente para o tratamento é acentuada se o terapeuta é flexível nos seus limites, sendo mais disponível ao cliente durante períodos de crise"; "a terapia tende a ser benéfica se uma forte aliança de trabalho é estabelecida e mantida durante o curso do tratamento"; "uma atitude de cuidado, cordialidade e aceitação tende a facilitar a mudança terapêutica" (Castonguay \& Beutler, 2006b).

Em meio às controvérsias em torno do que deveria ser empiricamente validado na pesquisa em psicoterapia e bastante preocupada com a lacuna ainda existente entre ciência e prática, a APA instaurou, em 2005, uma força-tarefa composta por psicólogos de diferentes abordagens com o objetivo de delinear um paradigma que fosse representativo de diversas perspectivas teóricas, metodológicas, conceituais e práticas (American Psychological Association, 2006). Assim, a APA validou o papel vital da pesquisa científica, a utilidade dos manuais de tratamento, a relevância das 
técnicas específicas, a importância dos fatores comuns, etc. (Norcross et. al., 2006).

Em vista disso, a APA desenvolveu o conceito de prática baseada em evidências em Psicologia (PBEP), definido como o processo individualizado de tomada de decisão clínica que ocorre por meio da integração da melhor evidência disponível com a perícia clínica no contexto das características, cultura e preferências do cliente (American Psychological Association, 2006). Os três componentes da definição - evidências de pesquisa, repertório do clínico e idiossincrasias do cliente - possuem o mesmo grau de importância na determinação da melhor conduta para cada cliente.

O primeiro componente da definição melhor pesquisa disponível - refere-se às evidências empíricas que demonstram quais procedimentos terapêuticos produzem resultados positivos e minimizam resultados negativos (Reed et al., 2006). Tendo em vista que o que constitui a melhor evidência depende do objetivo em questão, a APA (2006) referenda diferentes tipos de métodos nomotéticos e idiográficos (ensaios clínicos randomizados, experimentos de caso único, estudos de caso), que eventualmente podem culminar na listagem de tratamentos empiricamente sustentados (Chambless et al., 1998), relações terapêuticas empiricamente sustentadas (Norcross, 2002) e princípios de mudança terapêutica (Castonguay \& Beutler, 2006b).

A expressão melhor evidência disponível sugere a existência de dados com diferentes níveis de qualidade, ficando a cargo do terapeuta a seleção dos mais relevantes. Neste caso, o valor das evidências é estimado com base em dois critérios: (1) grau de compatibilidade das evidências com o caso em questão e (2) grau de confiabilidade dessas evidências, determinada pela quantidade de pesquisas e pela qualidade metodológica das mesmas. É fato que os dados de pesquisa podem ser incompatíveis, conflitantes ou incompletos, mas, ainda assim, deve-se buscar o uso da melhor evidência disponível sob a premissa de que pouca evidência, se bem utilizada, é melhor do que nenhuma evidência (Spencer, Detrich \& Slocum, 2012).

A perícia clínica diz respeito ao repertório especializado do terapeuta, construído em sua formação acadêmica, supervisão, experiência clínica e estudo da literatura teórica e empírica. Esse repertório inclui competências como avaliação diagnóstica, formulação de caso, identificação de padrões clinicamente relevantes, planejamento e implementação de intervenções, monitoração do progresso, habilidades interpessoais, estabelecimento da relação terapêutica, compreensão das diferenças individuais e culturais, comunicação com outros profissionais envolvidos, domínio teórico e, em especial, obtenção e aplicação das melhores evidências disponíveis para cada caso particular. Dessa forma, deve haver interação constante entre o julgamento profissional e os dados empíricos existentes (American Psychological Association, 2006; Reed et al., 2006; Wampold, Goodheart \& Levant, 2007).

De acordo com Spring (2007), a perícia clínica é o componente mais controverso da definição de PBEP, sobretudo porque costuma ser erroneamente concebida como um conjunto de intuições particularizadas sobre quais ações do terapeuta produziriam ou não bons resultados. Alguns terapeutas defendem que dados de pesquisa não podem guiar a prática e que a escolha dos procedimentos terapêuticos deveria se basear fundamentalmente na intuição oriunda da experiência profissional, mas há um considerável corpo de evidências sugerindo que a intuição não é um bom guia; pelo contrário, a intuição costuma estar repleta de vieses, falhas e distorções, a ponto de o terapeuta enxergar sucesso quando há indícios de fracasso (Baron, 2000; Herbert, Neeren \& Lowe, 2007). Em contraponto à ideia de um julgamento clínico escorado apenas na opinião do terapeuta, a perícia clínica é concebida como um conjunto rigoroso de competências que complementa (e não rivaliza com) a melhor evidência disponível (Spencer et al., 2012; Wampold et al., 2007).

A racional do terceiro componente da definição de PBEP - contexto das características, 
cultura e preferências do cliente-é promover a participação dos clientes nas tomadas de decisão sobre sua própria saúde e bem-estar, uma vez que intervenções costumam ser mais bem-sucedidas quando levam em consideração as particularidades do indivíduo, tais como seus objetivos, valores, crenças, preferências, contexto sociocultural e estado clínico (American Psychological Association, 2006). A identificação das peculiaridades do cliente como um fator determinante para a escolha dos procedimentos envolvidos no processo terapêutico é central para a PBEP. Como exemplo, Wampold et al. (2007) afirmam que um terapeuta que deixa de analisar como as crenças religiosas do cliente estão relacionadas à intervenção oferecida não está praticando PBEP, assim como um terapeuta que ignora as evidências de pesquisa.

A articulação das preferências do cliente com os outros dois componentes da definição de PBEP é uma tarefa complexa. Por um lado, permitir que as preferências do cliente prevaleçam na tomada de decisão clínica sem levar em consideração os dados de pesquisa pode acarretar resultados insatisfatórios ou até prejudiciais, além de desperdício de tempo e de dinheiro. Por outro lado, atentar somente para as evidências empíricas sem levar em consideração as escolhas do cliente pode conduzir a intervenções com baixo nível de aceitação, colocando em risco sua efetividade. Em vista disso, é responsabilidade do terapeuta descrever os procedimentos a serem empregados, explicar sobre seus possíveis benefícios e riscos e advertir sobre intervenções alternativas (Spencer et al., 2012; Spring, 2007).

Conforme descrita anteriormente, a PBEP é caracterizada como um processo individualizado de tomada de decisão clínica que ocorre por meio da integração da melhor evidência científica com a perícia clínica e as idiossincrasias do cliente. Na prática, esse modelo costuma ser operacionalizado em cinco passos, a saber: (1) levantamento de questões clínicas; (2) busca pelas melhores evidências empíricas, principalmente em bancos de dados que são voltados para profissionais atuantes e não para pesquisadores e que, por isso, reúnem apenas revisões sistemáticas da literatura empírica, como o Turning Research Into Practice, o SUMSearch e a Cochrane Library; (3) apreciação crítica das evidências encontradas; (4) intervenção, embasada nas evidências e regulada pelo repertório do terapeuta e pelas características do cliente; (5) avaliação dos resultados da intervenção (Frueh, Ford, Elhai \& Grubaugh, 2012).

\section{Limites da Prática Baseada em Evidências} em Psicologia

Embora o modelo de PBEP represente um importante avanço para o desenvolvimento da Psicologia como ciência e profissão, seu status atual apresenta limitações importantes. Em primeiro lugar, a esmagadora maioria das publicações, incluindo os livros da APA (Goodheart et al., 2006; Norcross et al, 2006), focam-se quase que exclusivamente no modelo conceitual de PBEP e nas diversas controvérsias que circundam o tema, em detrimento do ensino das habilidades necessárias para sua aplicação. O contrário ocorre na medicina e na enfermagem, por exemplo, em que o desenvolvimento do repertório prático ocupa $60 \%$ ou mais dos livros sobre o tema, conforme análise de Walker e London (2007). Certamente, o desenvolvimento da Psicologia como ciência e profissão depende da aprendizagem de competências que permitam a aplicação da PBEP, tais como o uso efetivo das bases de dados que indexam artigos, avaliação crítica das pesquisas clínicas e revisões de literatura, domínio das técnicas terapêuticas, etc.

Além disso, uma importante deficiência da PBEP diz respeito à compreensão do que constitui uma evidência de boa qualidade. Para Lohr (2011), o modelo de PBEP concebido pela APA "ampliou o domínio de 'evidência' para incluir praticamente toda e qualquer forma de crença ou opinião clínica" (p. 100-101), o que enfraquece sua cientificidade. Nesse contexto, Stuart e Lilienfeld (2007) sugerem que o progresso da PBEP depende fortemente de três fatores: (1) melhor especificação acerca de qual tipo de dado oriundo de qual tipo de método realmente é uma evidência de qualidade; 
(2) identificação e interrupção do uso de terapêuticas nocivas (cf. Lilienfeld, 2007); (3) avaliação contínua da prestação de serviços psicoterápicos utilizando medidas de resultado objetivas. Tais ações podem fortalecer a PBEP, na medida em que "clarificam o rol de opções disponíveis para os terapeutas, ajudam a proteger os clientes de danos e oferecem a vantagem de permitir que os clínicos contribuam para o crescente corpo de conhecimento sobre o que funciona e o que não funciona na psicoterapia" (p. 616).

Outro limite da PBEP é a existência de diversos sistemas que se propõem a classificar se uma intervenção é ou não baseada em evidências, de modo que uma modalidade de psicoterapia pode ser considerada empiricamente sustentada em alguns deles e não em outros. Por exemplo, como apontam David e Montgomery (2011), não há concordância entre as diretrizes do National Institute for Health and Clinical Excellence (NICE), da Divisão 12 da APA e da American Psychiatric Association. Tal divergência, somada à controvérsia concernente à concepção de evidência científica, gera confusão entre psicólogos e clientes que visam utilizar as melhores terapêuticas disponíveis, além de ressaltar a necessidade de criação de um sistema unificado e cientificamente coeso.

Além das questões relativas à aprendizagem das habilidades necessárias para a PBEP, à vagueza do conceito de evidência e aos conflitos na classificação do estatuto científico das psicoterapias, o modelo de PBEP tem sido severamente criticado por ignorar se a teoria subjacente às técnicas terapêuticas sustentadas empiricamente dispõe de evidências ou é, pelo menos, plausível (e.g., David \& Montgomery, 2011; Lilienfeld, 2011). Como argumenta Lilienfeld (2011), "para separarmos o joio do trigo no campo da psicoterapia, não podemos avaliar pesquisa de processo ou de resultado no vácuo" (p. 110). Afinal, o mais importante passo do empreendimento científico é a construção, sustentação, modificação ou rejeição de teorias, que são a mais rigorosa e mais completa forma de conhecimento (Popper, 1963).
Do ponto de vista prático, o entendimento dos princípios teóricos que explicam os efeitos de uma intervenção é essencial para o aprimoramento da PBEP por, pelo menos, dois motivos. Em primeiro lugar, a compreensão dos princípios teóricos permite descrever a etiologia dos fenômenos clínicos, identificar os processos necessários para a melhora do quadro, adaptar os achados empíricos a cada caso individual, desenvolver novas técnicas terapêuticas e, em especial, otimizar a qualidade de intervenção por meio da ativação dos mecanismos de ação responsáveis pela mudança. Em segundo lugar, o entendimento da teoria subjacente à prática torna possível a exclusão de terapêuticas pseudocientíficas do rol de opções, sobretudo porque elas geralmente contêm alguns dos fatores comuns (e.g., empatia do terapeuta, crenças e expectativas do cliente, etc.) que podem levar a mudanças clinicamente significativas e, assim, se mostrarem mais eficazes do que nenhum tratamento, configurando-se como um tratamento empiricamente sustentando de acordo com os critérios da Divisão 12 da APA. Por exemplo, em razão dos fatores comuns, uma terapia de energização poderia se mostrar superior a um grupo controle sem nenhum tratamento em um ensaio clínico randomizado, embora tenha como premissa a suposição de que os problemas psicológicos são causados por bloqueios em campos de energia invisíveis, cuja existência nunca foi comprovada e é cientificamente implausível (Lilienfeld, 2011).

Devido à extrema relevância da teoria para a produção de conhecimento científico em psicoterapia, é possível concluir que o acúmulo de evidências empíricas sobre a eficácia de diferentes terapêuticas é insuficiente se não for acompanhado dos princípios teóricos que as fundamentam, posição defendida por diversos autores (e.g., David \& Montgomery, 2011; Kihlstrom, 2006; Lilienfeld, 2011, Lohr, 2011; Mattaini, 2007). Como adverte Kihlstrom (2006), a psicoterapia deve estar embasada no entendimento científico dos processos psicológicos assim como os tratamentos médicos são baseados no entendimento científico da anatomia e da fisiologia. 


\section{Considerações finais}

Tendo como ponto de partida a invalidação da utilidade da psicoterapia por Eysenck na década de 1950, o presente artigo apresentou o desenvolvimento histórico do conceito de PBEP, passando pela conclusão de que todas as psicoterapias são igualmente efetivas e pela discussão em torno do papel dos fatores comuns e dos fatores específicos no processo terapêutico, descrevendo o trabalho das diferentes forças-tarefa e o esforço da APA em elaborar um modelo representativo das diversas perspectivas teóricas, metodológicas e práticas, que, conforme visto, não é isento de problemas.

Apesar de suas limitações, a PBEP está no centro das atenções do cenário internacional da Psicologia e constantes esforços têm sido feitos nessa direção por pesquisadores e profissionais com o intuito de preencher a lacuna entre ciência e prática ainda existente na Psicologia Clínica. Espera-se que este artigo possa contribuir para uma maior participação da Psicologia brasileira nesse movimento. 
Referências
Addis, M. E., \& Cardemil, E. V. (2006). Psychotherapy manuals can improve outcomes. In J. C. Norcross, L. E. Beutler, \& R. F. Levant (Orgs.), Evidence-based practices in mental health: Debate and dialogue on the fundamental questions (pp. 131-140). Washington, DC: American Psychological Association.

Addis, M. E., Wade, W. A., \& Hatgis, C. (1999). Barriers to dissemination of evidence-based practices: addressing practitioners' concerns about manual-based psychotherapies. Clinical Psychology: Science and Practice, 6(4), 430-441.

American Psychiatric Association (2013). Diagnostic and statistical manual of mental disorders: DSM-5. Arlington, VA: American Psychiatric Publishing.

American Psychological Association. (2006). Evidence-based practice in psychology: APA presidential task force on evidencebased practice. American Psychologist, 61(4), 271-285.

Baptista, M. N. (2010). Questões sobre avaliação de processos psicoterápicos. Psicologia em Pesquisa, 4(2), 109-117.

Barlow, D. H., Boswell, J. F., \& ThompsonHollands, J. (2013). Eysenck, Strupp, and 50 years of psychotherapy research: a personal perspective. Psychotherapy, 50(1), 77-87.

Baron, J. (2000). Thinking and deciding (3a ed.). Cambridge: Cambridge University Press.

Beutler, L.E.(1979). Towardspecificpsychological therapies for specific conditions. Journal of Consulting and Clinical Psychology, 47(5), 882-897.

(1998). Identifying empirically supported treatments: what if we didn't? Journal of Consulting and Clinical Psychology, 66(1), 113-120.

(2002). The Dodo bird is extinct. Clinical Psychology: Science and Practice, 9(1), 30-34.

Beutler, L. E., \& Johannsen, B. E. (2006). Principles of change. In J. C. Norcross, L. E. Beutler, \& R. F. Levant (Orgs.), Evidencebased practices in mental health: Debate and dialogue on the fundamental questions (pp. 226-234). Washington, DC: American Psychological Association.

Bohart, A. D., O'Hara, M., \& Leitner, L. M. (1998). Empirically violated treatments: disenfranchisement of humanistic and other psychotherapies. Psychotheraphy Research, $8(2), 141-157$.

Castonguay, L. G., \& Beutler, L. E. (2006a). Principles of therapeutic change: a task force on participants, relationships, and techniques factors. Journal of Clinical Psychology, 62(6), 631-638.

Castonguay, L. G., \& Beutler, L. E. (Orgs.). (2006b). Principles of therapeutic change that work. New York, NY: Oxford University Press.

Chambless, D. L. (1993). Task force on promotion and dissemination of psychological procedures: a report adopted by the Division 12 Board. Washington, DC: American Psychological Association. Recuperado em dia mês ano, de http://www.apa.org/ divisions/div12/est/chamble2.pdf

(2002). Beware the Dodo bird: The dangers of overgeneralization. Clinical Psychology: Science and Practice, 9(1), 13-16.

Chambless, D. L., Baker, M., Baucom, D. H., Beutler L. E, Calhoun K. S., Crits-Christoph, P.,... Woody, S. R. (1998). Update on empirically validated therapies, II. The Clinical Psychologist, 51(1), 3-16.

Chambless, D. L., \& Ollendick, T. (2001). Empirically supported psychological interventions: Controversies and evidence. Annual Review of Psychology, 52, 685-716.

Chambless, D. L., Sanderson, W. C., Shoham, V., Johnson, S. B., Pope, K. S., Crits-Christoph, P.,... McCurry, S. (1996). An update on empirically validated therapies. The Clinical Psychologist, 49(2), 5-18.

Cozolino, L. (2010). The neuroscience of psychotherapy: Healing the social brain (2a ed.). New York, NY: W. W. Norton.

David, D., \& Montgomery, G. H. (2011). The scientific status of psychotherapies: a new evaluative framework for evidence-based psychosocial interventions. Clinical Psychology: Science and Practice, 18(2), 89-99. 
DeRubeis, R. J., Brotman, M. A. \& Gibbons, C. J. (2005). A conceptual and methodological analysis of the nonspecifics argument. Clinical Psychology: Science and Practice, 12(2), 174-183.

Eysenck, H. J. (1952). The effects of psychotherapy: an evaluation. Journal of Consulting Psychology, 16, 319-324.

Fava, G. A., \& Mangelli, L. (2001). Assessment of subclinical symptoms and psychological well-being in depression. European Archive of Psychiatry and Clinical Neuroscience, 251 Suppl 2, II47-52.

Filippin, L. I., \& Wagner, M. B. (2008). Fisioterapia baseada em evidência: uma nova perspectiva. Revista Brasileira de Fisioterapia, 12(5), 432-433.

Frueh, B C., Ford, J. D., Elhai, J. D., \& Grubaugh, A. L. (2012). Evidence-based practice in adult mental health. In P. Sturmey, \& M. Hersen (Orgs.), Handbook of evidence-based practice in clinical psychology, volume two: adult disorders (pp. 3-14). Hoboken: John Wiley \& Sons.

Gaudiano, B. A., Brown, L. A., \& Miller, I. W. (2011). Let your intuition be your guide? Individual differences in the evidencebased practice attitudes of psychotherapists. Journal of Evaluation in Clinical Practice, 17(4), 628-634.

Goodheart, C. D., Kazdin, A. E., \& Sternberg, R. J. (Orgs.). (2006). Evidence-based psychoterapy: where practice and research meet. Washington: American Psychological Association.

Guyatt, G., Cairns, J., Churchill, D., Cook, D., Haynes, B., Hirsh, J.,... Tugwell, P. (1992). Evidence-based medicine: a new approach to teaching the practice of medicine. Journal of the American Medical Association, 268(17), 2420-2425.

Herbert, J. D., Neeren, A. M., \& Lowe, M. R. (2007). Clinical intuition and scientific evidence: what is their role in treating eating disorders. The Renfrew Perspective, Winter, 15-17.

Kazdin, A. E. (2005). Treatment outcomes, common factors, and continued neglect of mechanisms of change. Clinical Psychology: Science and Practice, 12(2), 184-188.

Kazdin, A. E., \& Bass, D. (1989). Power to detect differences between alternative treatments in comparative psychotherapy outcome research. Journal of Consulting and Clinical Psychology, 57(1), 138-147.

Kihlstrom, J. F. (2006). Scientific research. In J. C. Norcross, L. E. Beutler, \& R. F. Levant (Orgs.), Evidence-based practices in mental health: Debate and dialogue on the fundamental questions (pp. 23-31). Washington: American Psychological Association.

Koerner, N., Vorstenbosch, V., \& Antony, M. M. (2012). Panic disorder. In P. Sturmey, \& M. Hersen (Orgs.), Handbook of evidencebased practice in clinical psychology, volume two: adult disorders (pp. 285-311). Hoboken: John Wiley \& Sons.

Lilienfeld, S. O. (2007). Psychological therapies that cause harm. Perspectives in Psychological Science, 2, 53-70.

(2011). Distinguishing scientific from pseudoscientific psychotherapies: evaluating the role of theoretical plausibility, with a little help from reverend Bayes. Clinical Psychology: Science and Practice, 18(2), 105-112.

Lohr, J. M. (2011). What is (and what is not) the meaning of evidence-based psychosocial intervention? Clinical Psychology: Science and Practice, 18(2), 100-104.

Luborsky, L., Rosenthal, R., Diguer, L., Andrusyna, T. P., Berman, J. S., Levitt, J. T.,... Krause, E. D. (2002). The Dodo bird verdict is alive and well - mostly. Clinical Psychology: Science and Practice, 9(1), 2-12.

Luborsky, L., Singer, B. H., \& Luborsky, L. (1975). Comparative studies of psychotherapies: is it true that "everyone has won and all must have prizes"? Archives of General Psychiatry, 32(8), 995-1008.

Mattaini, M. A. (2007). The problem with evidence-based practice. Behavior and Social Issues, 16(2), 109-110.

Melnik, T., \&Atallah, A. N. (2011). Psicologia baseada em evidências: provas científicas da efetividade da psicoterapia. São Paulo: Gen/Santos. 
Melnik, T., Souza, W. F., \& Carvalho, M. R. (2014). A importância da prática da psicologia baseada em evidências: aspectos conceituais, níveis de evidência, mitos e resistências. Revista Costarricense de Psicología, 33(2), 79-92.

Morrow-Bradley, C., \& Elliott, R. (1986). Utilization of psychotherapy research by practicing psychotherapists. American Psychologist, 41(2), 188-197.

Norcross, J. C. (Org.). (2002). Psychotherapy relationships that work: Therapist contributions and responsiveness to patient needs. New York: Oxford University Press.

Norcross, J. C., Beutler, L. E., \& Levant, R. F. (Orgs.). (2006). Evidence-based practice in mental health: debate and dialogue on the fundamental questions. Washington, DC: American Psychological Association.

Reed, G. M., Kihlstrom, J. F., \& Messer, S. B. (2006). What qualifies as evidence of effective practice? In J. C. Norcross, L. E. Beutler, \& R. F. Levant (Orgs.), Evidencebased practices in mental health: Debate and dialogue on the fundamental questions (pp. 13-55). Washington, DC: American Psychological Association.

Richards, D. (2008). Reflecting the evidence. Evidence-Based Dentistry, 9(4), 98-99.

Rounsaville, B. J., \& Carroll, K. M. (2002). Commentary on dodo bird revisited: Why aren't we dodos yet? Clinical Psychology: Science and Practice, 9(1), 17-20.

Sanderson, W. C. (2003). Why empirically supported psychological treatments are important. Behavior Modification, 27(3), 290-299.

Sanderson, W. C. \& Woody, S. R. (1995). Manuals for empirically validated treatments: a project of the Task Force on Psychological Interventions. The Clinical Psychology, 48, 7-11.

Silva, G. A., \& Otta, E. (2013). Psicologia baseada em evidências: uma abordagem promissora a ser descoberta pelos psicólogos. Boletim Academia Paulista de Psicologia, 33(84), 20-29.

Smith, M. L., Glass, G. V., \& Miller, T. I. (1980). The benefits of psychotherapy. Baltimore, MD: John Hopkins University Press.
Sneed, J. R., Fertuck, E. A., Kanellopoulos, D., \& Culang-Reinlieb, M. E. (2012). Borderline personality disorder. In P. Sturmey, \& M. Hersen (Orgs.), Handbook of evidence-based practice in clinical psychology, volume two: adult disorders (pp. 507-529). Hoboken, NJ: John Wiley \& Sons.

Spencer, T. D., Detrich, R., \& Slocum, T. A. (2012). Evidence-based practice: A framework for making effective decisions. Education and Treatment of Children, 35(2), 127-151.

Spring, B. (2007). Evidence-based practice in clinical psychology: what it is, why it matters; what you need to know. Journal of Clinical Psychology, 63(7), 611-631.

Starcevic, V. (2003). Psychotherapy in the era of evidence-based medicine. Australasian Psychiatry, 11(3), 278-281.

Stiles, W. B., Hurst, R. M., Nelson-Gray, R., Hill, C. E., Greenberg, L. S., Watson, J. C.,... Hollon, S. D. (2006). What qualifies as research on which to judge effective practice? In J. C. Norcross, L. E. Beutler, \& R. F. Levant (Orgs.), Evidence-based practices in mental health: debate and dialogue on the fundamental questions (pp. 57-130). Washington DC: American Psychological Association.

Stirman, S. W., DeRubeis, R. J., CritsChristoph, P., \& Brody, P. E. (2003). Are samples in randomized controlled trials of psychotherapy representative of community outpatients? A new methodology and initial findings. Journal of Consulting and Clinical Psychology, 71(6), 963-972.

Strupp, H. H., \& Howard, K. I. (1992). A brief history of psychotherapy research. In D. K. Freedheim (Orgs.), History of psychotherapy: A century of change (pp. 309-334). Washington, DC: American Psychological Association.

Strupp, H. H. (1964). The outcome problem in psychotherapy: a rejoinder. Psychotherapy: Theory, Research and Practice, 1(3), 101.

Stuart, R. B., \& Lilienfeld, S. O. (2007). The evidence missing from evidence-based practice. American Psychologist, 62(6), 615-616. 
Task Force on Promotion and Dissemination of Psychological Procedures. (1995). Training in and dissemination of empirically-validated psychological treatments: report and recommendations. The Clinical Psychologist, 48(1), 3-23.

Walker, B. B.. \& London, S. (2007). Novel tools and resources for evidence-based practice in psychology. Journal of Clinical Psychology, 63(7), 633-642.

Wampold, B. E. (2010). The research evidence for the common factors models: A historically situated perspective. In L. B. Duncan, S. D. Miller, B. E. Wampold, \& M. A. Hubble (Orgs.), The heart and soul of change: delivering what works in therapy (2a ed.; pp. 49-81). Washington, DC: American Psychological Association.

Wampold, B. E., Goodheart, C. D., \& Levant, R. F. (2007). Clarification and elaboration on evidence-based practice in psychology. American Psychologist, 62(6), 616-618.
Westen, D., \& Arkowitz-Westen, L. (1998). Limitations of Axis II in diagnosing personality pathology in clinical practice. American Journal of Psychiatry, 155(12), 1767-1771.

Westen, D. I., Stirman, S. W., \& DeRubeis, R. J. (2006). Are research patients and clinical trials representative of clinical practice? In J. C. Norcross, L. E. Beutler, \& R. F. Levant (Orgs.), Evidence-based practices in mental health: Debate and dialogue on the fundamental questions (pp. 161-189). Washington, DC: American Psychological Association.

Williams, M., Powers, M. B., \& Foa, E. B. (2012). Obsessive-compulsive disorders. In P. Sturmey, \& M. Hersen (Orgs.), Handbook of evidence-based practice in clinical psychology, volume two: Adult disorders (pp. 313-335). Hoboken, NJ: John Wiley \& Sons.

Woody, S. R., \& Sanderson, W. C. (1998). Manuals for empirically supported treatments: 1998 update. The Clinical Psychologist, 51(1), 17-21. 


\section{Jan Luiz Leonardi}

Doutorando pela Universidade de São Paulo, São Paulo - SP. Brasil.

E-mail: janleonardi@gmail.com

\section{Sonia Beatriz Meyer}

Docente da Universidade de São Paulo, São Paulo - SP. Brasil.

E-mail: sbmeyer@usp.br

\section{Endereço para envio de correspondência:}

Rua Cardoso de Almeida, 977 - apto 151. CEP: 05013-001. São Paulo - SP.

E-mail: janleonardi@gmail.com

Recebido: 12/11/2014, Aprovado: 03/11/2015. 